\title{
Las maternidades juveniles en dos programas sociales en Argentina y México. Sentidos y prácticas institucionales en discusión *
}

\author{
ANA CECILIA GAITÁN** \\ CAROLINA GARCÍA CEJUDO ${ }^{* * * *}$
}

\begin{abstract}
Resumen
A partir de los avances de dos investigaciones cualitativas realizadas en Argentina y México, este trabajo presenta discusiones sobre las interacciones y las construcciones de sentido, en torno a la maternidad juvenil que se producen en las implementaciones de programas de inclusión dirigidos a mujeres jóvenes. Recuperando aportes de estudios feministas acerca de las regulaciones estatales sobre el género, esta investigación busca comprender cómo las prácticas y discursos de los/ as agentes estatales configuran regímenes institucionales de género y cómo estos se imbrican con la distribución de roles, atributos y jerarquías y con otros clivajes identitarios como la clase social y la edad.
\end{abstract}

Palabras clave: Política social - Mujeres jóvenes - Maternidad

\begin{abstract}
From accountings of two current qualitative researches from Argentina and Mexico, this paper submits some discussions about interactions and meaning production in inclusive program implementations focused to young women. With theoretical contributions from feminist studies of state regulations of gender, here are setted some analytic and interpretative guidelines to identify gender regimes from practices and discourses. To do so, it is problematized the gender social relations attending to the role distribution, attributes and hierarchies in conjuction with age and social class cathegories.
\end{abstract}

Key words: Social policy - Young mothers - Motherhood

Gaitán, Ana Cecilia y Cejudo, Carolina García "Las maternidades juveniles en dos programas sociales en Argentina y México. Sentidos y prácticas institucionales en discusión", en Zona Franca. Revista del Centro de Estudios Interdisciplinario sobre Mujeres, Año XXII, № 23, 2014, pp. 47-57.

Recibido: 12 de agosto 2014 - Aceptado: 2 de octubre 2014 


\section{Introducción}

ste artículo presenta los avances de la puesta en diálogo de dos investigaciones de doctorado respecto de las regulaciones estatales de género sobre la maternidad y sexualidad juvenil, ambas aún en curso. ${ }^{1}$ Circunscribiendo el análisis al caso del Conurbano Bonaerense, ${ }^{2}$ Argentina y al de Guanajuato, México, el propósito de este trabajo conjunto es abonar a la comprensión de las construcciones sobre la maternidad juvenil en contextos de implementación de políticas sociales en América Latina, atendiendo a las formas en que aquellas son atravesadas por el género, articuladamente con otros clivajes identitarios. Para ello, se recuperan los aportes de teóricas feministas que han caracterizado al Estado como fragmentado, conformado por múltiples capas y sitios de control y resistencia y a las acciones estatales como contradictorias entre sí (Haney, 1996). El Estado concebido como heterogéneo e intérprete se constituye como otro espacio de lo social donde se disputan las maneras en que se producen múltiples discursos, se interpretan necesidades y consecuentemente, se construyen las identidades de los sujetos sociales a lo largo de tales procesos (Fraser, 1989 y 1991 y Haney 2002). Tanto el aspecto reflexivo de los/ as agentes, como el carácter contestado de las acciones estatales y la capacidad de reapropiación y resistencia de los/as destinatarios/as de las políticas sociales, devienen aspectos centrales en el análisis de las re-significaciones locales y microsociales de los programas sociales. Desde esta perspectiva, los/as agen- tes estatales intervienen en las orientaciones de las acciones de la política social, dado que inciden en su implementación más allá de las disposiciones programáticas. Sus creencias y representaciones influyen en el despliegue de las intervenciones estatales (Goodwin, 1997 en Rodríguez Gustá, 2008).

Así las cosas, recuperando esta conceptualización feminista del Estado como una instancia de múltiples capas (Haney, 1996; Haney, 2002), compuesta por entramados de relaciones entre ejecutores/as y destinatarios/as de políticas sociales, este artículo analizará las implementaciones locales de dos políticas sociales destinadas a jóvenes a la luz del concepto de regímenes institucionales de género (Haney 1996). Tal como lo planteara Rodríguez Gustá (2008), recuperar dicho concepto supone abandonar la visión del Estado como ente homogéneo y uniforme, para comenzar a pensarlo como una trama de relaciones complejas y concretas con sus destinatarios/ as. Son las ideologías de las instituciones locales, las redes territoriales y las biografías de los/as agentes estatales, las que determinan la emergencia de dichos regímenes institucionales de género.

Atendiendo a la consideración teórica de que, para demostrar la existencia de regímenes institucionales de género, es preciso realizar un análisis más minucioso y extenso que de cuenta de los diferentes niveles analíticos que configuran dicha conceptualización, este artículo pretende identificar, a partir del análisis de algunos discursos y prácticas de los/as agentes estatales sobre la maternidad de las jóvenes, ciertos aspectos y atributos de posibles regímenes institucionales de género emer- gentes en nuestros campos investigativos.

\section{Dos políticas sociales para jóvenes}

Este artículo analiza las implementaciones de un programa de inclusión social juvenil en un barrio de sectores populares del conurbano bonaerense en Argentina y la de un programa de becas (transferencias condicionadas) para la inclusión educativa de jóvenes madres en dos municipios de Guanajuato, México.

El programa de Responsabilidad Social Compartida Envión comienza como una experiencia municipal destinada a la inclusión social y a la promoción de los derechos de adolescentes y jóvenes de entre 12 y 21 años en situación de "vulnerabilidad social". ${ }^{3}$ De acuerdo a la documentación oficial, sus objetivos son integrar a estos/as jóvenes al sistema educativo, enseñarles oficios, proveerles un espacio de contención y recreación a través de la oferta de talleres y actividades deportivas, culturales, lúdicas y artísticas. El grupo de trabajadores/as del programa es muy heterogéneo en cuanto a edades, historias, trayectorias educativas, laborales y de militancia. ${ }^{4}$

La ejecución del programa es municipal lo cual lleva a que cada implementación adquiera su particularidad de acuerdo al municipio en el que se encuentre anclada. Esta investigación analiza una de las implementaciones del Programa Envión en un municipio ubicado en el primer cordón del conurbano bonaerense. La decisión de circunscribir el trabajo de campo a aquel municipio estuvo vinculada al interés suscitado frente a la centralidad de la perspectiva de derechos para las políticas 
públicas municipales (Llobet et al., 2013). Tal como lo mencionaran otras investigaciones sobre políticas sociales en aquel municipio (Rodríguez Gustá, 2012 y Di Marco et al., 2006) el discurso de derechos sociales se encuentra presente, de manera evidente, en las distintas áreas de gobierno del mismo.

El Programa Becas de Apoyo a la Educación Básica de Madres Jóvenes y Jóvenes Embarazadas (Promajoven) es un programa compensatorio de becas que se propone para "contribuir a la reducción del rezago educativo mediante el otorgamiento de becas a niñas y jóvenes en contexto y situación de vulnerabilidad agravada por el embarazo y la maternidad". El presupuesto y su planteamiento se asigna desde el nivel de gobierno Federal y se designa que la implementación se realice a través de un consejo integrado por instancias de los gobiernos locales. El estudio se delimita a dos municipios en el contexto de las características particulares del estado de Guanajuato.

\section{Las tramas de lo particular: las implementaciones locales de los programas}

Cómo y quiénes validan o dan por autorizadas las interpretaciones de necesidades de las madres jóvenes y sus hijo/as, son algunos de las interrogantes que guiaron nuestra mirada en el análisis de la trama institucional (Fraser, 1991). Por ello, fue relevante identificar quiénes y desde qué lugar institucional se interpretan las necesidades. Tanto en el programa Envión como en Promajoven, la práctica concreta de la entrega de la transferencia se convierte, para algunas de ellas, en una mediación para ampliar su espectro de interacción con una red múltiple de instituciones, programas y niveles de gobierno.

Si bien el Envión no es un programa destinado a madres jóvenes, durante el trabajo de campo, el flujo de jóvenes embarazadas y con hijos/as que circulaban por la sede resultaba significativo. En la indagación se conoció, por un lado, que las agentes estatales del Envión compartían momentos de interacción y sociabilidad con las jóvenes madres, que rebasaban en tiempo y espacio de sus actividades formales y por el otro, que dichas jóvenes, como parte del "acuerdo compromiso" asumido con el programa, asisten semanalmente a un Taller de Educación Maternal Comunitaria ${ }^{5}$ como contraprestación de la beca económica recibida. Dicho taller es una política social dependiente de la Dirección de Educación del municipio, pero que, en el barrio considerado, su implementación se da de manera articulada con el programa Envión. EI TEMC surge en el año 2003 y se implementa en diversos barrios de sectores populares del municipio. Las destinatarias de la política son mujeres embarazadas y/o con hijos hasta los tres años que, por diversos motivos, no asisten al jardín maternal. El equipo de trabajo está conformado por trabajadoras sociales, psicólogas y docentes de educación inicial y maternal que, a diario, llevan a cabo el taller en los distintos barrios del municipio. De acuerdo a las pautas formales, este taller propone una mirada comunitaria de la educación maternal, siendo la finalidad del dispositivo repensar con las madres formas de disminuir cualquier riesgo en la primera infancia y el desarrollo integral de los niños/as. Se constituye como uno de los ejes de trabajo, revisar junto con las madres las pautas de crianza.
Debido a la preocupación municipal respecto del alto número de adolescentes embarazadas en el barrio analizado, desde el 2011 todas las semanas se realiza en la sede del Envión, un encuentro de dicho taller, destinado, exclusivamente, a madres jóvenes. La forma de convocatoria inicial en este barrio fue a partir de la información local provista por el programa Envión.

Agentes de ambas políticas sociales trabajan de manera conjunta: las docentes del TEMC participan de las reuniones de planificación de tareas y agenda, que mantienen los/as trabajadores/as del programa Envión, y estos/as últimos/as se encargan de ir, horas antes de que se inicie el taller, a "convocar" -llamar por teléfono y/o ir a buscar a las jóvenes a sus casas o dónde sea que estén- a las jóvenes para que, efectivamente, asistan. Ambos programas son lugares en los que, madres jóvenes intercambian opiniones e inquietudes respecto de la maternidad y la crianza con otras personas adultas que se encuentran por fuera de lo demarcado como "doméstico". Estos adultos se configuran, no sin tensiones, como sujetos habilitados para el intercambio de consejos sobre la crianza, pero también, sobre otros temas como ser: beneficios sociales, familias, parejas y sexualidad. Cabe destacar que, estos dos dispositivos, no son los únicos espacios estatales por los que circulan las jóvenes madres.

Promajoven-Guanajuato busca asignar el mayor número de becas y no hay casos de chicas que no se aceptan, por dos principales motivos, según se identifica; primero, porque la cantidad de becas que se otorga desde el presupuesto federal, es significativamente menor a la proporción de jóvenes del esta- 
do de Guanajuato que cumplen con el perfil para ser destinatarias del Promajoven; ${ }^{6}$ segundo, por la forma que interpretan la entrega de la beca por parte de funcionarios de gobierno y actores educativos. Aunque las coordinadoras de Promajoven no sostienen la idea que la entrega de las becas incentive entre las jóvenes la maternidad, existe resistencia por parte de algunas autoridades educativas y operadores de programas estatales a aceptar la existencia y difusión del programa. ${ }^{7}$ Por tanto, se percibe como un logro de efectividad en la implementación por parte de las instancias de Guanajuato, el hecho que se entreguen todas las becas asignadas al estado. Este reconocimiento se expresa al interior de Educafin pero también desde la autoridad federal que observa eficiencia en su gestión y "no pierde" presupuesto por becas no asignadas como en el caso de otros estados del país. ${ }^{8} \mathrm{La}$ gestión de difundir la convocatoria y comunicar la aceptación de las solicitantes la hace Educafin para todos los municipios de Guanajuato. Sin embargo, una vez que se transfiere el financiamiento a la cuenta de Educafin, las coordinadoras de Promajoven, que están en diferentes instancias, designadas según cada gestión municipal, son quienes tienen contacto directo con las becarias y entregan cara a cara el financiamiento. La recolección de información se realizó en dos municipios urbanos de Guanajuato que aquí se nombran como Del Apóstol y Del Río. ${ }^{9}$ En el primero, donde se ubica la sede central de Educafin, las coordinadoras entregan el cheque de la beca a las destinatarias dentro de las instalaciones. En el municipio Del Río se entrega en las instalaciones de la sede municipal del Sistema para el Desarrollo Integral de la Familia (DIF). Este momento, -la entrega del financiamientose identifica como el segundo contacto imprescindible entre las destinatarias (en adelante becarias) y el espacio institucional estatal en el contexto de la implementación del Promajoven. A partir de entonces se registra una diferenciación entre la gestión de los diferentes municipios; incluso dentro del mismo municipio Del Río existen diferencias respecto a la dinámica de las interacciones entre las becarias y las instancias estatales: en la parte céntrica de la ciudad principal del municipio Del Río existe un barrio (en adelante Los Eucaliptos) con una subsede del DIF municipal que se distingue por sus características.

\section{La multiplicidad de discursos y de modelos de maternidad}

El Envión y el Promajoven articulan, en la enunciación de sus objetivos programáticos, interpretaciones de necesidades. Pero ni la definición del perfil de las destinatarias, ni los objetivos, pueden ser leídos como las únicas fuentes de discursos y medios configuradores de regímenes institucionales de género (Haney, 1996). Si bien, en ambos programas es relevante la importancia de los discursos expertos en la definición del modo en el que se interviene, no se agota en ellos el espacio de interpretación de la intervención. En este sentido, tal como fuera mencionado, las creencias y representaciones de los/as agentes también son elementos que influyen en la concreción de las intervenciones (Goodwin 1997, citado en Rodríguez Gustá 2012) y en la configuración de regímenes institucionales de género (Haney 1996) particulares. Por lo tanto, las intervenciones estatales y sus discursos a través de Envión y Promajoven no se encuentran orientadas hacía una única dirección.

Para el caso del Envión, sus trabajadores/as consideran lo "vincular" como un aspecto "clave" de su labor definida, por ellos/as, como de una fuerte implicación laboral y afectiva (Gaitán, 2014). Tal como lo plantean, los/as jóvenes "se abren" a contarles situaciones personales muy sensibles y complejas y que no se animarían a comentar en sus propias familias. Además de las actividades grupales, los/as agentes se encargan del seguimiento personal de las/os jóvenes en cuanto a: participación en el programa, asistencia a la escuela, vínculos familiares, relación con pares y "parejas", "consumos problemáticos", maternidades, entre otros. En esta relación que se entabla, a través de entrevistas individuales -formales e informales- y actividades grupales de diverso tipo, resulta medular la experiencia. La centralidad de la experiencia del/la joven se expresa en la posibilidad de contar "todo" en espacios colectivos e individuales: de hacer pública su vida (Llobet et al., 2013). En el caso de los/as agentes también, pero desde el consejo y la autoreferencia (Gaitán, 2014). En cuanto al trabajo con las jóvenes madres se traduce en la escucha -incluso fuera del horario y del espacio asignado a las actividades del programa; la emisión de "consejos"; la distribución de ciertos beneficios que integran las políticas de tipo compensatorio y el otorgamiento de información sobre jardines maternales de la zona y atención médica. Subyacentes a estos consejos -a veces pueden acercarse a mandatos-que circulan de manera oral, operan ideologías muy distintas unas de 
otras, lo cual dificulta la posibilidad de pensar al conjunto de intervenciones desde el Envión, como homogéneo y unívoco. Estas maternidades juveniles son construidas y reconstruidas por las/os agentes, no sin tensiones y disidencias. En este sentido, parte de estas agentes caracterizan la maternidad de las destinatarias como problemática: las jóvenes "dejan su vida por el bebé”. En otras palabras, para estas agentes, las destinatarias dejarían de pensarse como mujeres jóvenes, para comenzar a concebirse sólo como madres, traducido en el descuido físico, la ausencia en actividades del programa y el abandono de los estudios, entre otras cosas. Su intervención hacía estas jóvenes se encuentra orientada a generar en ellas autonomía y mayor independencia en relación a sus hijos/as, estableciéndose determinadas expectativas para estas jóvenes sobre todo en relación a sus vínculos con sus niños/as y parejas. Despliegan consejos, en oportunidades cuestionamientos, respecto de aquellas relaciones. Se les suele aconsejar que entren en diálogo y construyan un "buen vínculo" con los padres de sus hijos/as, con el objeto de construir una crianza compartida y dividida equitativamente en tareas (desde el pago de cuotas alimentarias hasta los cuidados diarios) lo cual permitiría que todas las responsabilidades no recaigan ni sobre las jóvenes ni sobre los otros miembros femeninos de la familia. Tal como fuera mencionado, la experiencia de las agentes resulta un elemento central en sus intervenciones. Es por ello que, cuando abordan la relación de estas destinatarias con los padres de sus hijos/as, las agentes recuperan su propia experiencia con sus parejas, para ponerla en diálogo con la de las jóvenes (Gaitán, 2014). En este sentido, la expectativa central de este grupo de agentes es que las jóvenes puedan convertirse en sujetos autónomos. Para algunas de aquellas, la apuesta a construir jóvenes madres autónomas es descrita en clave de empoderamiento. Parte de este empoderamiento está asociado a la planificación de un proyecto de vida "más pensante": que al igual que ellas, las destinatarias puedan planear en qué momento ser madres y lograr establecer un vínculo con los padres de sus hijos/as que les permita continuar con otros aspectos de sus vidas como mujeres. A la vez que, aprendan a demandar beneficios estatales. Para lograr que sus intervenciones sean exitosas despliegan, articuladamente con otras políticas sociales locales, provinciales y nacionales, toda una serie de estrategias ${ }^{10}$ que tornan, por momentos, confusos los límites entre las administraciones. Todas estas estrategias desplegadas, desde las más cotidianas y sencillas, hasta las que requieren mayor planificación, se encuentran orientadas a que las jóvenes construyan un "proyecto de vida" que les permita, entre otras cosas, planificar el próximo embarazo.

Así las cosas, cuando las destinatarias se embarazan, la intervención se encuentra dirigida a "acompañar" a la joven. La cercanía forjada configura, en muchas oportunidades, a las agentes como las primeras adultas a las que las destinatarias acuden al conocer su embarazo. De ahí en adelante, por un tiempo, las agentes se convierten en confidentes (Gaitán, 2014). Como confidentes no sólo acompañan a las jóvenes a la realización de consultas y exámenes médicos sino también, durante la comunicación del embarazo entre sus familiares y parejas (instancia descrita por las agentes como de mucha tensión). Este "apoyo", que suele ser relatado como una situación conflictiva, es caracterizado como incondicional. Una de las agentes, al recordar con angustia el momento de acompañar a una joven a una consulta para obtener información sobre la interrupción del embarazo (con la que la agente estaba en desacuerdo), relató: "Bueno, la decisión es de ella. Nosotras la íbamos a apoyar en la decisión que ella hacía, la íbamos a acompañar" (Operadora barrial).

Muchas de las agentes estatales se encuentran de acuerdo con el aborto ${ }^{11}$ y manifiestan su interés porque el mismo se legalice en el país, mientras que otras, como la del relato anterior, si bien acompañan a las jóvenes al centro de salud barrial para acceder a información sobre prácticas abortivas seguras, ${ }^{12}$ debido a la cercanía en el vínculo y la confianza tramada, se expresan en desacuerdo con la posibilidad de interrupción del embarazo. Estas últimas agentes consideran la maternidad como algo más asociado a lo instintivo y natural, como algo para lo que y con lo que se nace. Sus intervenciones, como en el caso de las otras agentes, se encuentran centradas en el consejo y la referencia, a la vez que se sostienen en determinadas expectativas para las destinatarias. Estas intervenciones se encuentran atravesadas por la clasificación de estas destinatarias en dos grupos, uno de madres más desatendidas y otras más cuidadosas. Tal como lo expresa una de estas agentes, dentro de las jóvenes madres que transitan por el programa están aquellas que son un "tiro al aire" y otras que son "re mamás" (agente estatal). 
En este sentido, parte de las expectativas de la intervención de estas agentes se encuentra orientada a trabajar y transformar al grupo de mamás "que dejan sus chicos tirados por ahí, que andan por ahí", en aquellas otras madres que "re cuidan a sus hijos" (agente estatal). Tal como lo mencionara una operadora barrial respecto a su trabajo con una de las destinatarias:

"Clara, vos tenés que cuidarlo porque tu hijo es tu hijo, vos lo tenés que cuidar, él tiene que estar limpito, arregladito, dado de comer, bien, bien"-le digo- "y si yo te llego a ver con el nene sucio, no sabés cómo cobras vos!" le digo, ¿viste?. Porque también, tuvimos quejas con ella de que llevaba a los chicos sucios, ¿viste a la guardería?, Clara”. (...)Me llamó la directora, me encontró un día ella y me dice: "¿conocés a Clara?" "Sí, Clara" -le digo- "va al Envión". "Uy, yo quisiera hablar con ustedes" -me dice- "porque a esa chica le falta contención, alguien que le enseñe, que cumpla con los horarios, porque..." $Y$ yo: "sí, va a la guardería a cualquier hora, que, era las 12 y quería que, que entre la guardería al nene" viste. ${ }^{13}$

Parte de las jóvenes madres destinatarias del Envión también participan del TEMC. Cada encuentro del taller supone la realización de diversas actividades, desde lúdicas y recreativas, hasta de "exploración" -trabajo de estímulo de sentidos y de conocimiento del cuerpo de los/as niños a través de la experimentación con témperas, pinturas, comida, música, etc- y conversaciones que van desde lactancia hasta narración y confección de cuentos, entre otros. Tras la actividad central se desarrolla un momento final del taller, que es el de la merienda. ${ }^{14}$ Durante esta última instancia se producen charlas informales entre las jóvenes y entre éstas y las docentes donde circulan consejos sobre parejas, familias, educación y salud -incluyendo aseo y alimentación- entre otros temas. Para las docentes del TEMC, el vínculo entre las jóvenes madres y sus hijos/as, suele encontrarse distendido, motivo por el cual, sus intervenciones están destinadas a que aquellas "se involucren" con la crianza. Si bien desde los objetivos programáticos, las docentes son comprendidas como educadoras populares y el espacio de taller, como una instancia de aprendizaje mutuo, se ha observado que en la intervención concreta, aparecen operando ideologías moralizadoras y modeladoras de las conductas de esas jóvenes y cierta tensión entre los objetivos del TEMC y los sentidos que las agentes estatales le otorgan a esta maternidad. En este sentido, estas agentes presentan a las destinatarias, no sin ambigüedad, otro modelo de crianza que consideran más positivo, de acuerdo a la crianza inadecuada recibida por estas jóvenes de pequeñas. Si bien estas agentes encuentran a la maternidad como algo instintivo y natural, como "algo para lo que ellas mismas han nacido" y "con lo que se nace", cuando estas se refieren a las madres jóvenes de sectores populares, la "función materna universal" pareciera no ser tan obvia:

"Acá hay mamás muy chicas también y cuanto más chicas son las mamás, menos experiencia tienen en muchas cosas. Yo a veces me planteaba y digo, pensar que una a veces viene con cosas que, que ya lo trae la mujer, ¿no? como eso de la maternidad, es como que hay cosas que te salen. Pero, yo me doy cuenta, que hay chicas que no, que ni siquiera lo tienen, o sea, no tienen el sentido común de sermamás, o sea, necesitan que alguien las guíe o, o ver qué es lo que pueden hacer $[\ldots]^{\prime 15}$

Por un lado, si bien el instinto maternal es caracterizado como algo instintivo, en el caso de estas jóvenes madres de sectores populares, aquel debe ser guiado de manera racional para no traer aparejado consecuencias negativas: "una mamá que no desea amamantar, o que lo hace sin seguir las pautas establecidas como correctas, no forjará de manera adecuada el vínculo con su hijo/a" (docente TEMC). Estas representaciones que las agentes tienen sobre la maternidad juvenil en sectores populares implican determinadas expectativas no sólo respecto del vínculo de estas jóvenes con sus hijos/as, sino también, con las familias y el barrio. Sus expectativas de la intervención las giran en torno del reforzamiento del vínculo madre-hijo/a -que se supone laxo- $y$ al fortalecimiento de pautas de crianza monoparentales. En este sentido, la intervención se esfuerza en establecer límites entre quiénes se encuentran habilitados para la crianza y quiénes no. Se fortalece el protagonismo de la madre en la crianza de sus hijos y la pertinencia del consejo "experto" para las maternidades jóvenes, supuestamente carentes de instinto y desdibujadas por las dinámicas barriales y familiares. Así, las docentes del TEMC parecen recuperar formulaciones respecto de la crianza, coincidentes con las dominantes, aplicándolas en este contexto particular.

En la forma con la que se plantea el Promajoven y en algunos rasgos de su implementación, visto en el cuadro de lo nacional, quedan en relevancia algunos discursos. Primeramente el rasgo individualizante característico de ideologías 
sobre poblaciones que se asumen en minoridad (en este caso mujeres y menores de edad) (Llobet, 2012). Se asume que el embarazo y la maternidad es responsabilidad de las jóvenes y sus familias, sin reconocer la importancia y relación de la joven con su contexto. Con ello, se ocultan las omisiones del ámbito estatal y del contexto respecto a la privación ya evidente del acceso a sus derechos básicos (educación, salud, educación sexual, bienestar y condiciones favorables de vida). Cabe resaltar que Promajoven se plantea como un programa compensatorio, asumiendo la pérdida de lo que implica haber interrumpido los estudios y esto, planteado con relación directa al embarazo o la maternidad. Cosa que, al menos en los municipios de Guanajuato estudiados, dista de ocurrir tal relación en esa dirección pues sólo algunas de las becarias interrumpieron sus estudios por el embarazo; la mayoría lo hizo por eventos ajenos a su maternidad. En segundo lugar, destaca en el planteamiento del Promajoven la interpretación sobre el cuidado infantil. Se otorga una beca para que la joven continúe sus estudios pero no se articula política pública o proceso institucional alguno que responda a la necesidad del cuidado infantil de los/ as hijo/as de las jóvenes.

Para la consecución del objetivo del Promajoven el cuidado infantil se interpreta en general, de poca relevancia y sin connotación política. El esfuerzo para terminar los estudios y para criar al hijo/a no es una práctica que quede en el ámbito de lo social sino en lo individual y lo doméstico. El hecho de que no haya instancia alguna de cuidado infantil para lo/as hijo/as de las jóvenes y que no se haga siquiera el planteamiento de la posibilidad de guarderías para que ellas asistan al sistema escolar de asistencia diaria y regular, denota rasgos del discurso maternalista y familiarista. Se parte de las ideas que la mujer se debe ocupar del cuidado infantil basado en la equivalencia que se asigna a la relación de ser madre y ser mujer, además que se prioriza al espacio de lo doméstico y la familia respecto a su involucramiento. En efecto, se omite la mención de la responsabilidad y presencia de los padres o parejas de los hijos de las jóvenes, dejando de lado la responsabilidad del progenitor o de la pareja que pueda tener la joven. El Programa no considera en sus requisitos condicionalidad alguna sobre el estado civil o las características vinculares entre las jóvenes y sus parejas.

Sin embargo, incluso ante estos grandes rasgos, se pueden identificar otros discursos a través de las prácticas institucionales implementadas y a través de las interpretaciones de los agentes estatales. En primer lugar, el hecho que Promajoven sea planteamiento de una instancia educativa a nivel federal (Secretaría de Educación Pública: SEP) pero dirigida a los contextos locales en sus múltiples posibilidades de gestión (y no ligada obligatoriamente a instancias estatales de la Secretaría de Educación), es una de las condiciones de posibilidad para que las becarias se sitúen en un espacio que permita varias posibilidades en las formas de relaciones entre ellas y los agentes estatales, abriendo posibilidades de interpretación en la construcción cotidiana de los sentidos de la maternidad. Esto posibilita que los momentos en que se hacen operativas y se traducen en decisiones burocráticas alguna de las interpretaciones, se produzca un marco de flexibilidad para que se presenten disputas, resistencias y cruce de diferentes discursos, haciéndose visible en la dinámica misma de las prácticas institucionales.

Promajoven en Guanajuato presenta una interacción peculiar primeramente entre las mismas instancias gubernamentales y en segundo término, entre las interacciones de las becarias y otras instancias estatales. Los momentos que más posibilitan la interacción con varios niveles y ámbitos de gobierno son: (1) la difusión de la convocatoria, (2) el seguimiento de los requisitos del Promajoven y (3) la convocatoria a otros eventos no relacionados directamente con Promajoven.

Durante estos tres momentos se registra diferenciación en algunas características de gestión del Promajoven Guanajuato respecto a otros estados. Respecto a la difusión de la convocatoria, por ejemplo, las coordinadoras reportan que donde existe mayor presencia de diversidad étnico-cultural y donde la maternidad infanto-juvenil es una práctica buscada y esperada por las tradiciones locales, el Promajoven se difunde sin mayor restricción al interior de las aulas y pasillos escolares de las secundarias. En esas características, el esfuerzo de las coordinadoras Promajoven se concentra en la selección de las solicitantes en lugar de la búsqueda de captación de solicitantes.

La información de la convocatoria al Promajoven para captar becarias se difunde cuando es de manera directa, principalmente a través del contacto que los asesores educativos del INEA o INAEBA tienen con las jóvenes y hay que resaltar que esto ocurre una vez que ya están fuera del sistema educativo formal y que optaron por volver a 
retomar sus estudios básicos. En el Municipio Del Apóstol las becarias provienen principalmente por la referencia que se da del programa en el INAEBA. Aunque se registran becarias Promajoven que cursan en INEA, en este municipio no se observó una participación activamente intencional. Otra forma de difusión es a través de la búsqueda directa de mamás jóvenes en los hospitales del Centro de Salud, donde regularmente instalan un stand informativo.

La convocatoria Promajoven se difunde indirectamente a través de las presentaciones que realizan las coordinadoras del programa de Educafin a cuantas instancias sean posibles. De estas, relatan diferentes formas de aceptación y colaboración, que implican una interpretación peculiar sobre la entrega de dinero a mujeres jóvenes estudiantes con hijos. Para el caso del municipio Del Apóstol no hay una colaboración estrecha con el DIF estatal o las autoridades educativas estatales, sin embargo sí con el Instituto donde difunden directamente la información.

Las coordinadoras Promajoven Del Apóstol se oponen al supuesto que las becas incentivan a las jóvenes a embarazarse y que éstas impliquen la promoción de un "mal ejemplo". Ellas consideran que asumirlo implica una posición prejuiciosa, que ignora las carencias con las que viven las jóvenes y no reconoce las intenciones de las becarias para buscar mejores condiciones de vida. Resalta su inconformidad con la forma que otras instancias de gobierno en Guanajuato interpretan Promajoven, a tal grado que las coordinadoras consideran que -de tener las posibilidades- buscarían "hacer conciencia" e incidir en las formas de mirar el programa y la maternidad juvenil. ${ }^{16}$
Se identifica una diferencia importante entre la orientación de las coordinadoras de Promajoven que tienen contacto directo con las becarias respecto a los demás agentes estatales. ${ }^{17}$ Éstas expresaron que las becarias se apoyan en sus propias familias para el cuidado de sus hijos y que sienten impotencia en su intervención porque la mayoría de las becarias no son conscientes del proceso que están viviendo. Expresaron la importancia que ellas "agarren la onda", "despierten", "reaccionen" para hacerse cargo de sus decisiones y terminar sus estudios al mismo tiempo que cuidan a sus hijos. Las coordinadoras que han tenido este contacto directo refirieron a momentos donde intervinieron al margen de lo que les corresponde institucionalmente, asumiendo una responsabilidad ajena a sus lugares institucionales. Resulta ilustrativa su intervención cuando detectaron un caso en el que se estaba prostituyendo a una joven que había quedado embarazada y que solicitó la beca Promajoven:

"la niña dejó de estudiar porque la mamá la prostituía. Nosotros fuimos a buscar apoyo, a hablar con ella. EI DIF nos pidió apoyo a nosotros cuando, pues ellos como DIF tienen todo un sistema, de violencia, atención psicológica y tienen como más herramientas para ir y meter una denuncia. Sin embargo, nosotros fuimos".

Las interpretaciones en torno a los diferentes aspectos de la problematización de la maternidad juvenil son diferentes entre los asesores educativos del INEA e INAEBA. Todos consideraron que significaba un problema a evitar, una situación indeseable que habría que prevenir. Sin embargo, sólo algunos relacionaron el contexto económico y social de las chicas como dimensiones importantes de tal "problema". Hubo otros que además de colocar la maternidad de las jóvenes en un plano paralelo y en asociación a la drogadicción y el suicidio, las culpabilizaron evidenciando un discurso patologizador e individualista sobre la maternidad de las jóvenes.

Finalmente, cabe destacar que para las coordinadoras Promajoven del municipio Del Río, el objetivo del Programa se plantea en un sentido diferente.

Ellas enunciaron que con el programa se trata de evitar que se embaracen de nuevo, más allá de que estudien (o no): "que no reincidan en el embarazo" y "que se auto-gestionen como mujeres" -expresó una de ellas. En efecto, al reconocer este otro sentido de la entrega de la beca en el contexto de otras prácticas institucionales (como talleres posnatales, interacción entre las becarias mientras asisten a eventos de propaganda local y cursos de diversos temas), se derivan en sus supuestos, diferentes interpretaciones sobre la maternidad y su problematización respecto a las coordinadoras del Municipio Del Apóstol.

\section{Consideraciones finales}

La indagación de las implementaciones locales del Envión y el Promajoven ha permitido iluminar y significar aspectos específicos de las prácticas institucionales y las interpretaciones de los/as agentes estatales respecto a la maternidad para el caso de jóvenes madres de sectores populares. Se ha dado cuenta cómo las expectativas de la intervención de las/os agentes hacen que las prácticas y los mensajes que las sostienen adquieran aspectos propios que 
las dotan de singularidad, no sólo respecto de las representaciones sobre las relaciones de las jóvenes con sus familias, el barrio y el Estado, sino también, de las soluciones y respuestas brindadas. En este sentido, el análisis de ambos casos da cuenta de cómo las capacidades interpretativas y relacionales del Estado se despliegan diferencialmente aún en un mismo territorio (Rodríguez Gusta, 2008).

En suma, los avances aquí presentados permiten pensar, tanto para el caso de Buenos Aires como para el de Guanajuato, que las intervenciones estatales sobre las maternidades juveniles distan de ser unívocas en cada caso, abonando así a los desarrollos feministas que han concebido al Estado como heterogéneo y con múltiples capas de gobierno que pueden ser inconsistentes y hasta contradictorios entre sí. Se ha visibilizado también que estos programas sociales destinados a jóvenes de sectores populares en sus implementaciones locales entran en contacto, y hasta se implementan de manera articulada, con otros programas y espacios estatales no necesariamente correspondientes al mismo nivel o sector de gobierno. En este contacto y articulación entre programas, se despliega una multiplicidad discursiva a través de los/as agentes estatales, quienes influyen en las orientaciones de las acciones de política social, incidiendo en su implementación más allá de las disposiciones programáticas (Llobet et al., 2013). Tal como lo explicara Haney (1996), y derribando la idea del Estado como estructura que impone unilateralmente un set singular de normas de género a las destinatarias, las instituciones locales, las redes territoriales y las biografías de las/os agentes que implementan las políticas inciden en la emergencia de diferentes atributos y características de modelos discursivos que podrían estar dando cuenta de la existencia de diversos regímenes institucionales de género.

Por último, dado que este artículo se ha centrado en el análisis de las prácticas y los discursos de los/as agentes estatales, queda introducida y abierta la pregunta sobre las significaciones y re-significaciones realizadas por las destinatarias acerca de las ideologías sobre la maternidad en el marco de las implementaciones de los programas sociales. En este sentido, identificar la circulación de diferentes discursos y posibilidades de interacción entre las destinatarias y el espacio estatal, habilita para profundizar en la comprensión de las disputas, tensiones y resistencias sobre la construcción de la maternidad y por tanto, las regulaciones estatales de género.

Notas:

* Este artículo tiene como antecedentes dos ponencias presentadas en el XXXII Congreso Internacional de la Asociación de Estudios Latinoamericanos LASA y en la I Bienal Latinoamericana de Infancias y juventudes. Democracias Derechos Humanos y Ciudadanías.

** Ana Cecilia Gaitán es Antropóloga por la Universidad de Buenos Aires (UBA) y doctoranda en Antropología Social (UBA). Desarrollada su tesis de doctorado con una Beca de Posgrado otorgada por el Consejo Nacional de Investigaciones Científicas y Técnicas (CONICET), la cual se encuentra radicada en el Instituto Interdisciplinario de Estudios de Género (IIEGE-UBA). También forma parte del Programa de Estudios Sociales en infancias y juventud de Universidad Nacional de San Martín (UNSAM).ce_gaitan@yahoo.com.ar

*** Carolina García Cejudo es socióloga, maestra en ciencias de la educación (UAQ) y doctoranda del Programa de Ciencias Sociales de la Facultad Latinoamericana de Ciencias Sociales (FLACSO), Sede Académica Argentina. Forma parte del Programa de Estudios Sociales en infancias y juventud de Universidad Nacional de San Martín (UNSAM).candoragc@yahoo.com

1. Ambas investigaciones se enmarcan en el Programa de Estudios Sociales en Infancias y Juventud de la Universidad Nacional de San Martín (UNSAM).

La investigación llevada a cabo en Buenos Aires es de corte etnográfico. Como parte del trabajo de campo en la implementación de la política social, se realizó observación participante en diversos espacios relevantes de la misma (tanto en actividades cotidianas como instancias particulares); entrevistas en profundidad y semi-estructuradas a agentes estatales, funcionarios/as y destinatarias. También se trabajó con el relevamiento de fuentes secundarias: documentos e información interna sobre el programa, como así también con normativas y disposiciones per- 
tinentes. Para el caso de la investigación desarrollada en Guanajuato se realizó un acercamiento con aproximación etnográfica. Se revisó archivo de expedientes de becarias, se aplicaron fichas de observación participante y se realizaron entrevistas estructuradas y semiestructuradas tanto individuales como grupales a becarias, Coordinadoras de Promajoven, autoridades educativas y operadores de los programas relacionados.

2. Se denomina conurbano bonaerense a un aglomerado de veinticuatro partidos del Gran Buenos Aires, dispuestos en cordones de acuerdo con la cercanía de la Ciudad Autónoma de Buenos Aires (Maceira, 2012).

3. Para este programa son adolescentes y jóvenes vulnerables "aquellos que pertenecen a hogares con inserción laboral precaria, los que no estudian ni trabajan, viven en situaciones familiares de violencia y abandono, residen en viviendas precarias, en barrios con infraestructura y equipamiento inadecuado".

4. Más sobre los/as trabajadores/as del programa y la legitimación de sus prácticas Llobet et al. 2013 y Gaitán, 2014.

5. De ahora en más TEMC.

6. Para el 2010 , el padrón del programa reporta la asignación de 298 becas para todo el estado de Guanajuato, mientras que en los datos del Conteo del 2005 se identifican para esta entidad, 2,226 mujeres con características a los que se dirige el Promajoven (en edad escolar -de 12 a 18 años- con al menos un hijo vivo y con estudios incompletos del nivel básico). Padrón de la población atendida por entidad federativa, Municipio y edad para agosto 2010. Programa de becas de apoyo a la educación básica de madres jóvenes y jóvenes embarazadas. Subsecretaría de Educación Básica. Dirección General de Educación Indígena. Dirección de Educación Básica. Estadística INEGI. II Conteo de Población y Vivienda 2005. Población de 12 a 18 años de edad.

7. Entrevista informal a Directora de Plaza Comunitaria (2011). Entrevista informal a Coordinadora Promajoven en Municipio Del Apóstol (2012). Entrevista a autoridad educativa de la Secretaría de Educación de Guanajuato (2013).

8. Entrevista grupal a Coordinadoras del Promajoven estatal en Educafin (2013). Entrevista informal a Directora de Promajoven en la Subsecretaría de Educación Básica. Dirección General de Educación Indígena. Dirección de Educación Básica (2011).

9. Los nombres de los municipios se omiten intencionalmente y se asignaron nombres ficticios, procurando el anonimato de los actores entrevistados e involucrados durante la recolección de información.

10. Talleres temáticos; introducir la pregunta por el deseo o no del embarazo; ayuda en la obtención de controles ginecológicos; la entrega de anticonceptivos; el cuidado de sus hijos para que las jóvenes participen en las actividades de Envión; acuerdos con escuelas de la zona para que las jóvenes puedan terminar el colegio; la negociación de vacantes en jardines de infantes, facilitación del acceso de beneficios sociales y la exigencia de que las jóvenes lleven un registro escrito de sus actividades y obligaciones, entre otras.

11. El aborto en Argentina solo es no punible en los casos en que se encuentre en peligro la vida o salud de la mujer embarazada y cuando el embarazo provenga de una relación sexua no consentida. Ver www.adc.org.ar/2013/wp-content/ uploads/2013/12/Aborto-NP-protocolos-comparados-ADC-dic2013.pdf y www. abortolegal.com.ar.

12. Las Consejerías Pre y Post aborto son espacios donde se brinda escucha e información a las mujeres cursando un embarazo no planificado. Si la mujer decide continuar con su embarazo, se le realizan todos los controles preparto necesarios. Si resuelve lo contrario, y no se enmarca en un aborto no punible, se le transmite información sobre riesgos y daños producidos por ciertas prácticas abortivas, a la vez que, se les provee información validada científicamente y de carácter público acerca de prácticas seguras. Se explican pautas de alarma y se les indica las guardias hospitalarias a las que recurrir de producirse eventuales complicaciones. Durante la consulta post aborto se le ofrece todos los métodos anticonceptivos disponibles. En el barrio donde fue realizado el trabajo de campo la consejería funciona en el Centro de Atención Primaria de Salud, constituyéndose como una política pública municipal.

13. Entrevista a operadora Barrial del programa Envión (2013)

14. Comida ligera que se toma a media tarde $o$ antes de la cena.

15. Entrevista grupal a docentes del TEMC (2013). 
16. "que se organicen campañas de comunicación dirigidas especialmente a los funcionarios y encargados de programas para que se comprenda mejor el problema de la maternidad en las jóvenes" (Entrevista grupal a Coordinadoras Promajoven).

17. Estos son: asesores educativos de los sistemas de educación abierta INEA e INAEBA, trabajadoras sociales del DIF municipal y las autoridades estatales educativas.

\section{Referencias bibliográficas}

BALDERAS, Ronaldo. (2009), "Guanajuato ha encarcelado a 130 mujeres por aborto: Las Libres", El Correo de Guanajuato, 17.

DI MARCO, Graciela; Rodríguez Gustá, A. L.; LLobet. V. y Brener, A. (2006). "EI Plan Jefes y Jefas de Hogar: una mirada desde los procesos de ampliación de derechos de las mujeres". En Cuartas Jornadas de Investigación en Antropología Social. Buenos Aires, Argentina.

FRASER, Nancy. (1991). "La lucha por las necesidades: esbozo de una teoría crítica socialista-feminista de la cultura política del capitalismo tardío". Debate Feminista, 3, 3-40.

FRASER, Nancy. (1989). "Talking about Needs: Interpretive Contests as Political Conflicts in Welfare-State Societies". Ethics, 99(2), 291.

GAITÁN, Ana Cecilia. (2014) "Algunas reflexiones sobre la construcción cotidiana de la maternidad y la sexualidad de jóvenes madres, en la implementación de una política social en el conurbano bonaerense". Revista de Estudios Sociales, $N^{\circ} 49,47-58$.

HANEY, Lynne. (2002). Inventing the needy: Gender and the politics of welfare in Hungary. Berkeley: University of California Press.

HANEY, Lynne. (1996). "Homeboys, babies, men in suits: The state and the reproduction of male dominance". American Sociological Review. 759-778.

LLOBET, Valeria., Gaitán C., Medan, M. y Magistris G. (2013) “'Este espacio es para que ustedes hablen'. La legitimación de la intervención en los programas sociales". En Llobet V. (coord.) Sentidos de la exclusión social. Beneficiarios, necesidades y prácticas en políticas sociales para la inclusión de niños/as y jóvenes. Buenos Aires: Biblos. Serie investigaciones y ensayos. 129-259

LLOBET, Valeria. (2012). "Políticas sociales y ciudadanía: Diálogos entre la teoría feminista y el campo de estudios de infancia". Frontera Norte, 24(48), 7-36.

MACEIRA, Verónica. (2012) "Notas para una caracterización del Área Metropolitana de Buenos Aires" en Boletín Informativo. Observatorio das Metropoles. Año III, Número 224.

RODRÍGUEZ G., Ana Laura. (2012). "Capacidades institucionales y efectos de género: la implementación local de un programa de transferencia condicionada". Ponencias del IV Congreso de la Asociación Uruguaya de Ciencia Política. Montevideo: AUCIP.

RODRIGUEZ G., Ana Laura. (2008). Las políticas sensibles al género: variedades conceptuales y desafíos de intervención. Temas y Debates, (16), 109-129. 